Pacific Journal of Mathematics

SOME TOPOLOGICAL PROPERTIES OF SPACES OF 


\section{SOME TOPOLOGICAL PROPERTIES OF SPACES OF MEASURES}

\section{G. Koumouluis}

Let $X$ be a completely regular space and $M_{\tau}(X), M_{t}(X)$ and $M_{c}(X)$ the spaces of the $\tau$-additive measures, tight measures and measures with compact support on $X$, endowed with the weak topology. The aim of this paper is to study topological properties that devolve from $X$ to $M_{\tau}(X), M_{t}(X)$ and $M_{c}(X)$ or their positive cones $M_{t}^{+}(X), M_{t}^{+}(X)$ and $M_{c}^{+}(X)$. It is proved that if $X$ is paracompact (resp. Lindelöf) and Cech complete, then $M_{\tau}^{+}(X)$ and $M_{t}^{+}(X)$ have the same properties, but $M_{c}^{+}(X)$ does not (unless $X$ is compact). If $X$ is realcompact then $M_{c}(X)$ has the same property, but $M_{\tau}(X)$ and $M_{t}(X)$ need not. However, if $X$ is realcompact paracompact, then $M_{\tau}(X)$ is realcompact.

Let $X$ be a completely regular space and $C(X)$ the space of bounded real-valued continuous functions on $X$ with the supremum norm. The spaces $M_{\sigma}(X), M_{\tau}(X), M_{t}(X)$ and $M_{c}(X)$ of measures on $X$ are defined as subsets of the dual of $C(X)$ ([22]) and can be described using the Stone-Cech compactification of $X$ ([13]). Identifying each point $x$ of $X$ with the point mass $\varepsilon_{x}, X$ can be considered as a closed subset of $M_{s}(X)$ and $M_{s}^{+}(X)$ (for $s=\tau, t$ or $c$ ) endowed with the weak topology which is defined by $C(X)$. Our purpose is to find topological properties that devolve from $X$ to $M_{s}(X)$ or $M_{s}^{+}(X)$.

Notations and preliminary results are given in $\S 1$. In $\S 2$ it is proved that certain topological properties which can be described by perfect functions with values in metric spaces, devolve from $X$ to $M_{s}^{+}(X)$ but not to $M_{s}(X)$. For this purpose it is shown that a perfect function between two spaces $X$ and $Y$ induces a perfect function between $M_{s}^{+}(X)$ and $M_{s}^{+}(Y)$. Section 3 is concerned with the property of realcompactness. Using a result of Corson, it is proved that $M_{s}(X)$ is realcompact if and only if every $\sigma$-additive measure on $X$ which on every countably generated $\sigma$-algebra of Baire sets coincides with some element of $M_{s}(X)$ is, in fact, an element of $M_{s}(X)$. Realcompactness devolves from $X$ to $M_{c}(X)$, but not to $M_{\tau}(X)$ and $M_{t}(X)$

Finally, I wish to thank Professor S. Negrepontis for his assistance and encouragement and Dr. A. Tsarpalias for bringing [2] to my attention.

1. Preliminaries and notations, A basic reference for the 
theory of measures on topological spaces is Varadarajan [22]. All topological spaces considered in this paper are assumed to be completely regular (and, of course, Hausdorff). Let $X$ be a basic fixed completely regular space. We denote by $C(X)$ the space of bounded continuous real-valued functions on $X$, endowed with the supremum norm. A linear functional $\varphi$ on $C(X)$ is

(a) $\sigma$-additive, if $\varphi\left(f_{n}\right) \rightarrow 0$ for every sequence $\left\{f_{n}\right\}$ in $C(X)$ which is decreasing and pointwise convergent to zero;

(b) $\tau$-additive, if $\varphi\left(f_{\alpha}\right) \rightarrow 0$ for every net $\left\{f_{\alpha}\right\}$ in $C(X)$ which is decreasing and pointwise convergent to zero;

(c) tight, if $\varphi\left(f_{\alpha}\right) \rightarrow 0$ for every uniformly bounded net $\left\{f_{\alpha}\right\}$ in $C(X)$ which converges to zero uniformly on compact subsets.

According to the Aleksandrov representation theorem [22, Part I, Theorem 6], the dual of $C(X)$ is identified by an order preserving isometry with the space $M(X)$ of (finitely additive Baire) measures on $X$. A measure $\mu$ on $X$ is said to be $\sigma$-additive, $\tau$-additive or tight if the functional

$$
\varphi(f)=\int_{X} f d \mu, \quad f \in C(X)
$$

has these properties. The spaces of the $\sigma$-additive, $\tau$-additive and tight measures on $X$ are denoted by $M_{\sigma}(X), M_{\tau}(X)$ and $M_{t}(X)$ respectively. The elements of $M_{\sigma}(X)$ are exactly the countably additive measures defined on the $\sigma$-algebra $\mathscr{B}(X)$ of all Baire sets of $X$ (see [22, Part I, Theorem 18]). We recall that the collection of Baire sets of $X$ is the $\sigma$-algebra generated by the zero sets of real-valued continuous functions on $X$.

The space of measures with compact support is denoted by $M_{c}(X)$. The elements of $M_{c}(X)$ correspond to the functionals on $C(X)$ with the property: $\varphi\left(f_{\alpha}\right) \rightarrow 0$ for every net $\left\{f_{\alpha}\right\}$ in $C(X)$ which converges to zero uniformly on compact subsets. Usually we do not distinguish between the functional and the associated measure.

We have $M(X) \supset M_{o}(X) \supset M_{\tau}(X) \supset M_{t}(X) \supset M_{c}(X)$. The subsets of positive measures in these spaces are denoted by $M^{+}(X), M_{o}^{+}(X)$, $M_{*}^{+}(X), M_{t}^{+}(X)$ and $M_{c}^{+}(X)$.

If $\mu \in M(X), \bar{\mu}$ denotes the corresponding regular Borel measure on the Stone-Cech compactification $\beta X$ of $X$ (via the isometry of $C(X)$ and $C(\beta X))$.

Theorem 1.1 (Knowles [13]). If $\mu \in M^{+}(X)$, then

(i) $\mu \in M_{\sigma}^{+}(X)$ if and only if $\bar{\mu}$ vanishes on all zero sets of $\beta X$ contained in $\beta X-X$;

(ii) $\mu \in M_{:}^{+}(X)$ if and only if $\bar{\mu}$ vanishes on all compact sets contained in $\beta X-X$; 
(iii) $\mu \in M_{t}^{+}(X)$ if and only if for every $\varepsilon>0$ there is a compact set $K \subset X$ such that $\bar{\mu}(\beta X-K)<\varepsilon$.

A (nonzero) measure is 2 -valued if its range is $\{0,1\}$. The $\tau$ additive 2-valued measures on $X$ are exactly the point masses $\varepsilon_{x}$, $x \in X$.

On the space $M(X)$ we consider the weak topology $\sigma(M(X), C(X))$. This space is completely regular and can be identified with the space $M(\beta X)$ by the homeomorphism $\mu \rightarrow \bar{\mu}$. Also, the function $x \rightarrow \varepsilon_{x}$ is a homeomorphism of $X$ and $\left\{\varepsilon_{x}: x \in X\right\}$ which is closed in $M_{*}^{+}(X)$.

The following theorem is one of the deepest results obtained by Varadarajan in [22]. A simpler proof can be found in [5].

Theorem 1.2 ([22, Part II, Theorem 13]). The space $M_{:}^{+}(X)$ is metrizable if and only if $X$ is metrizable.

Using 1.2 Varadarajan also proves the following.

Theorem 1.3 ([22, Part II, Theorems 14, 18]). The space $M_{\tau}^{+}(X)$ is separable (resp. complete) metrizable if and only if $X$ is separable (resp. complete) metrizable.

Let $X, Y$ be completely regular spaces and $f: X \rightarrow Y$ a continuous function. We define $f_{*}: M(X) \rightarrow M(Y)$ by $f_{*}(\mu)(g)=\mu(g \circ f)$ for every $g \in C(Y)$. It is immediate that $f_{*}$ is continuous (with respect to the weak topologies $\sigma(M(X), C(X))$ and $\sigma(M(Y), C(Y)))$ and $f_{*}\left(M_{s}(X)\right) \subset$ $M_{s}(Y)$ for $s=\sigma, \tau, t$ or $c$. If $\mu \in M_{o}(X)$, then $f_{*}(\mu)$ coincides with the classical image measure, i.e.,

$$
f_{*}(\mu)(B)=\mu\left(f^{-1}(B)\right) \text { for all } B \in \mathscr{B}(Y)
$$

(cf. $[7$, p. 163] and $[19$, Theorem $3.1(\mathrm{c})]$ ).

2. Perfect functions. A continuous function $f: X \rightarrow Y$ is called perfect if $f$ is onto, closed and $f^{-1}(\{y\})$ is compact for every $y \in Y$. The following theorem will be used to show that some topological properties related to Cech completeness and $M$-property (defined below) devolve from $X$ to $M_{s}^{+}(X)$ for $s=\tau, t$ or $c$.

THEOREM 2.1. Let $f: X \rightarrow Y$ be continuous onto. Then, $f_{*}$ : $M_{s}^{+}(X) \rightarrow M_{s}^{+}(Y)$ (for $s=\tau, t$ or $c$ ) is perfect if and only if $f$ is perfect.

We shall use the following two lemmas. 
LEMmA 2.2. If $f: X \rightarrow Y$ is continuous, onto, then $f_{*}: M(X) \rightarrow$ $M(Y)$ is onto and $f_{*}\left(M^{+}(X)\right)=M^{+}(Y)$.

This is a well-known application of Hahn-Banach theorem.

Lemma 2.3. Let $f: X \rightarrow Y$ be a perfect function and $\mu \in M^{+}(X)$. If $f_{*}(\mu) \in M_{s}^{+}(Y)$ then $\mu \in M_{s}^{+}(X)$, for $s=\tau, t$ or $c$.

Proof. Let $\bar{f}: \beta X \rightarrow \beta Y$ be the continuous extension of $f$. We observe that $\overline{f_{*}(\mu)}=\bar{f}_{*}(\bar{\mu})$ and, by the uniqueness of the regular Borel extension of Baire measures on compact spaces, $\overline{f_{*}(\mu)}(B)=$ $\bar{\mu}\left(\bar{f}^{-1}(B)\right)$ for all Borel sets $B$ in $\beta Y$. We also note that $\bar{f}(\beta X-X) \subset$ $\beta Y-Y$ since $f$ is perfect (cf. [1, Lemma 9.2]).

Now assume that $f_{*}(\mu) \in M_{\tau}(Y)$. For every compact set $K \subset$ $\beta X-X$ we have $f(K) \subset \beta Y-Y$ and, by Theorem 1.1 (ii),

$$
\begin{aligned}
0 & =\overline{f_{*}(\mu)}(\bar{f}(K))=\bar{\mu}\left(\bar{f}^{-1}(\bar{f}(K))\right) \\
& \geqq \bar{\mu}(K) \geqq 0 .
\end{aligned}
$$

Therefore $\mu \in M_{\tau}^{+}(X)$.

The proof for $s=t$ or $c$ is similar.

Proof of Theorem 2.1. We have $f_{*}\left(M_{s}^{+}(X)\right) \subset M_{s}^{+}(Y)$, so $f_{*}$ : $M_{s}^{+}(X) \rightarrow M_{s}^{+}(Y)$ is well-defined.

Suppose that $f_{*}$ is perfect. Identifying $X$ with the closed subset $\left\{\varepsilon_{x}: x \in X\right\}$ of $M_{s}^{+}(X), f$ is the restriction of $f_{*}$ to this set, hence it is perfect.

Conversely, assume that $f$ is perfect. Clearly $f_{*}: M_{s}^{+}(X) \rightarrow M_{s}^{+}(Y)$ is continuous and Lemmas 2.2 and 2.3 combine to show that $f_{*}$ is onto.

To show that $f_{*}$ is closed let $F$ be a closed subset of $M_{s}^{+}(X)$, $\left\{\mu_{\alpha}\right\}$ a net in $F$ and $\nu \in M_{s}^{+}(Y)$ such that $f_{*}\left(\mu_{\alpha}\right) \rightarrow \nu$. We have $\left\|\mu_{\alpha}\right\|=\mu_{\alpha}(1)=f_{*}\left(\mu_{\alpha}\right)(1) \rightarrow \nu(1)$. Now by Alaoglu-Bourbaki theorem $\left\{\mu_{\alpha}\right\}$ has a cluster point $\mu \in M^{+}(X)$. The continuity of $f_{*}$ implies $f_{*}(\mu)=\nu$ and, by Lemma 2.3, $\mu \in M_{s}^{+}(X)$. Since $F$ is closed in $M_{s}^{+}(X)$ we have $\mu \in F$ and $\nu \in f_{*}(F)$. Therefore $f_{*}(F)$ is closed in $M_{s}^{+}(Y)$.

It remains to show that $f_{*}^{-1}(\{\nu\})$ is compact for every $\nu \in M_{s}^{+}(Y)$. Clearly $f_{*}^{-1}(\{\nu\})$ is closed in $M_{s}^{+}(X)$ and by Lemma 2.3 it is closed in $M^{+}(X)$. For every $\mu \in f_{*}^{-1}(\{\nu\})$, we have $\|\mu\|=\mu(1)=f_{*}(\mu)(1)=\nu(1)$, so $f_{*}^{-1}(\{\nu\})$ is norm bounded. It follows that $f_{*}^{-1}(\{\nu\})$ is compact.

REMARK. Theorem 2.1 is not correct for the function $f_{*}: M_{s}(X) \rightarrow$ $M_{s}(Y)$, as simple examples show that $f_{*}^{-1}(\{\nu\})$ need not be norm bounded. Also, Theorem 2.1 is not correct for $s=\sigma$. Indeed, by [23, p. 189] there is a perfect function $f$ from a realcompact space 
$X$ onto a space $Y$ which is not realcompact. Then the set $F=$ $\left\{\varepsilon_{x}: x \in X\right\}$ is closed in $M_{\sigma}^{+}(X)$, but $f_{*}(F)=\left\{\varepsilon_{y}: y \in Y\right\}$ is not closed in $M_{\sigma}^{+}(Y)$.

A space $X$ is Cech complete if $X$ is a $G_{\delta}$ set in $\beta X$. According to a theorem of Frolik (cf. [1, Theorem 9.4]), a space $X$ is paracompact and Cech complete if and only if there is a perfect function from $X$ onto a complete metric space. It follows that $X$ is Lindelöf and Cech complete if and only if there is a perfect function from $X$ onto a separable complete metric space. Using these results, Varadarajan's metrization theorem and Theorem 2.1 we have the following.

Corollary 2.4. The space $M_{s}^{+}(X)$ (for $s=\tau$ or $\left.t\right)$ is

(i) paracompact and Cech complete, or

(ii) Lindelöf and Cech complete, if and only if $X$ has property (i) or (ii) respectively.

Proof. First we observe that for a Cech complete space $X$ we have $M_{\tau}(X)=M_{t}(X)$ since $X$ is a Borel set in $\beta X$ (cf. [13, Theorem 3.4]). If $X$ has property (i) (resp. (ii)), then, by the above, there is a perfect function $f$ from $X$ onto a complete (resp. separable complete) metric space $Y$. By Theorem 2.1, the function $f_{*}: M_{\tau}^{+}(X) \rightarrow$ $M_{\tau}^{+}(Y)$ is perfect, where $M_{\tau}^{+}(Y)$ is complete (resp. separable complete) metrizable space (Theorem 1.3). It follows that $M_{\tau}^{+}(X)=M_{t}^{+}(X)$ is paracompact (resp. Lindelöf) and Cech complete.

The converse follows from the fact that $X$ is homeomorphic to a closed subset of $M_{s}^{+}(X)$.

A space $X$ is called an $M$-space if there is a quasi-perfect function from $X$ onto a metric space. (A continuous function $f: X \rightarrow Y$ is quasi-perfect if $f$ is onto, closed and $f^{-1}(\{y\})$ is countably compact for every $y \in Y$ ). According to a theorem of Morita a space $X$ is a paracompact $M$-space if and only if there is a perfect function from $X$ onto a metric space (see Morita [18] and the reference given there for the theory of $M$-spaces). It follows that $X$ is a Lindelö $M$-space if and only if there is a perfect function from $X$ onto a separable metric space. Analogously we have the following.

Corollary 2.5. The space $M_{s}^{+}(X)$ (for $s=\tau, t$ or $c$ ) is

(i) paracompact $M$-space, or

(ii) Lindelöf $M$-space, if and only if $X$ has property (i) or (ii) respectively. 
The following proposition which appears in Gröming [6] gives information about the topological properties which devolve from $X$ to $M_{s}^{+}(X)$, for $s=\tau$ or $t$. For the sake of completeness we include a proof which is shorter than that of Grömig.

Proposition 2.6. The space $X^{N}$ is homeomorphic to a closed subset of $M_{:}^{+}(X)$ which is contained in $M_{t}^{+}(X)$.

Proof. We define

$$
h:(\beta X)^{N} \rightarrow M^{+}(\beta X) \quad \text { by } \quad h\left(\left(x_{n}\right)_{n \in N}\right)=\sum_{n=1}^{\infty} \frac{1}{2^{n}} \cdot \varepsilon_{x_{n}} .
$$

It is clear that $h$ is $1-1$ and $h\left(X^{N}\right) \subset M_{t}^{+}(X)$.

To see that $h$ is continuous, let $\left\{\left(x_{n}^{\alpha}\right)_{n \in N}\right\}_{\alpha \in A}$ be a net in $(\beta X)^{N}$ and $\left(x_{n}\right)_{n \in N} \in(\beta X)^{N}$ such that $x_{n}^{\alpha} \rightarrow x_{n}$ for every $n \in N$. Let $\mu_{\alpha}=$ $h\left(\left(x_{n}^{\alpha}\right)_{n \in N}\right)$ and $\mu=h\left(\left(x_{n}\right)_{n \in N}\right)$. We shall show that $\mu_{\alpha} \rightarrow \mu$. Clearly $\mu_{\alpha}(\beta X) \rightarrow \mu(\beta X)$ and by [22, Part II, Theorem 2], it is enough to show that $\lim \inf \mu_{\alpha}(U) \geqq \mu(U)$ for every cozero set $U$ of $\beta X$. Let $C=\left\{n \in N: x_{n} \in U\right\}$. For every finite set $F \subset C$ there is $\alpha_{F^{*}} \in A$ such that $x_{n}^{\alpha} \in U$ for all $\alpha>\alpha_{F}$ and $n \in F\left(\right.$ since $x_{n}^{\alpha} \rightarrow x_{n}$ ). Then

$$
\mu_{\alpha}(U) \geqq \sum_{n \in F} \frac{1}{2^{n}} \quad \text { for all } \alpha>\alpha_{F} .
$$

Therefore

$$
\lim \inf \mu_{\alpha}(U) \geqq \sum_{n \in F} \frac{1}{2^{n}} \text { for all finite } F \subset C,
$$

that is, $\lim \inf \mu_{\alpha}(U) \geqq \sum_{n \in C} 1 / 2^{n}=\mu(U)$.

Since $(\beta X)^{N}$ is compact, $h$ is a topological embedding. Moreover, $h\left(X^{N}\right)$ is contained in the compact set $h\left((\beta X)^{N}\right)$, therefore

$$
\operatorname{cl}_{M_{\tau}^{+}(X)}\left(h\left(X^{N}\right)\right) \subset M_{\tau}^{+}(X) \cap h\left((\beta X)^{N}\right)=h\left(X^{N}\right) .
$$

The last equality follows from Theorem 1.1(ii). So $h\left(X^{N}\right)$ is closed in $M_{\tau}^{+}(X)$ and this finishes the proof.

REMARK. Every finite product of $X$ is homeomorphic to a closed subset of $M_{\tau}^{+}(X)$ which is contained in $M_{c}^{+}(X)$. Indeed, for every $m \in N$, we define

$$
h_{m}:(\beta X)^{m} \rightarrow M^{+}(\beta X) \quad \text { by } \quad h_{m}\left(\left(x_{i}\right)_{1 \leqq i \geqq m}\right)=\sum_{i=1}^{m} \frac{1}{2^{i}} \varepsilon_{x_{i}} .
$$

Then $h_{m}\left(X^{m}\right) \subset M_{c}^{+}(X)$ and we can proceed as in the proof of Proposition 2.6 . 
As Grömig remarked in [6, p. 401], Proposition 2.6 and the above remark yield that if $P$ is a topological property which is hereditary on closed subsets and devolves from $X$ to $M_{\tau}^{+}(X)$ or $M_{t}^{+}(X)$ (resp. $M_{c}^{+}(X)$ ), then $P$ devolves from $X$ to the countable (resp. finite) products of $X$. Moreover, we know that if $X$ is Lindelöf, then $X \times X$ may fail to be even normal. Thus Corollaries 2.4 and 2.5 are not correct for Lindelöf or paracompact spaces and it is not incidental that the topological properties considered in these corollaries devolve from $X$ to $X^{N}$. However, the topological properties of Corollary 2.4 as well as Cech completeness do not devolve from $X$ to $M_{c}^{+}(X)$ as the next proposition shows. Notice that Cech completeness is hereditary on closed subsets and devolves from $X$ to $X^{N}$ (see [3, pp. 144, 145]) and, more generally, from $X$ to $M_{\tau}^{+}(X)$ and $M_{t}^{+}(X)$ (cf. [22, Part II, Theorem 17]).

Proposition 2.7. $M_{c}^{+}(X)$ is paracompact (or Lindelöf) and Cech complete if and only if $X$ is compact.

Proof. If $X$ is compact, then $M_{c}^{+}(X)=M_{t}^{+}(X)$, so $M_{c}^{+}(X)$ has the desired properties.

For the converse, we show that if $M_{c}^{+}(X)$ is Cech complete, then $X$ is pseudocompact; this is sufficient because every pseudocompact paracompact space is compact.

Let $f: X \rightarrow \boldsymbol{R}$ be a continuous function. We define

$$
U_{n}=\{x \in X:|f(x)|>n\}
$$

and

$$
F_{n}=\left\{\mu \in M_{c}^{+}(X): \mu\left(U_{n}\right)=0\right\}
$$

for $n=1,2, \cdots$. Each $F_{n}$ is closed in $M_{c}^{+}(X)$ and $M_{c}^{+}(X)=\bigcup_{n=1}^{\infty} F_{n}$ because every compact subset of $X$ is contained in the complement of some $U_{n}$. Since every Cech complete space satisfies the Baire category theorem (see [3, p. 145]), some $F_{n_{0}}$ has nonempty interior. Then $U_{n_{0}}=\varnothing$ and $f$ is bounded. This finishes the proof.

REMARK. Cech completeness of $M_{c}^{+}(X)$ does not imply compactness of $X$. Indeed, if $W$ is the space of countable ordinals, then $W$ is locally compact and every $\sigma$-compact subset of $W$ is relatively compact. It follows that $M_{c}^{+}(W)=M_{t}^{+}(W)$ is Cech complete, although $W$ is not compact.

We finish this section by showing that the analogues of Corollaries 2.4 and 2.5 for $M_{s}(X)$ fail badly. The following lemma which is essentially due to Corson (cf. Lemma 4 and the proof of Lemma 9 in [2]) will be needed. 
LEMMA 2.8. Let $M$ be a vector subspace of $M(X)$. Then

(i) $M$ satisfies the countable chain condition; and

(ii) for every continuous function $f$ from $M$ into a separable metric space, there is a countable $K \subset C(X)$ such that

$$
\mu, \nu \in M,\left.\mu\right|_{K}=\left.\nu\right|_{K} \Longrightarrow f(\mu)=f(\nu) \text {. }
$$

(i) follows from the fact that $M$ is homeomorphic to a dense subset of $\boldsymbol{R}^{I}$ for some $I$ (take $I$ to be an algebraic basis of $C(X)$ ) and that $\boldsymbol{R}^{r}$ satisfies the countable chain condition. The arguments used in [20] to show that every continuous function from $\boldsymbol{R}^{I}$ into a separable metric space is determined by countably many coordinates apply for dense subsets of $\boldsymbol{R}^{T}$ and this proves (ii).

THEOREM 2.9. The following are equivalent for $s=\tau, t$ or $c$.

(i) $M_{s}(X)$ is an $M$-space;

(ii) $M_{s}(X)$ is Cech complete;

(iii) $X$ is finite.

Proof. (iii) $\Rightarrow$ (i) and (iii) $\Rightarrow$ (ii) are trivial.

(i) $\Longrightarrow$ (iii). There is a quasi-perfect function $F: M_{s}(X) \rightarrow Y$, where $Y$ is metrizable. Since $M_{s}(X)$ satisfies the countable chain condition (Lemma 2.8(i)), $Y$ satisfies the same condition, so it is separable. By Lemma 2.8 (ii), $F$ is determined by a countable set $K \subset C(X)$. The set

$$
L=\left\{\mu \in M_{s}(X): \mu(f)=0 \text { for all } f \in K\right\}
$$

is contained in the countably compact set $F^{-1}(F\{(0)\})$; also $\lambda \cdot \mu \in L$ for every $\lambda \in \boldsymbol{R}$ and $\mu \in L$. It follows that $L=\{0\}$. Therefore the function

$$
G: M_{s}(X) \longrightarrow \boldsymbol{R}^{K} \quad \text { with } G(\mu)=(\mu(f))_{f \in K}
$$

is 1-1 and continuous. We define

$$
H: M_{s}(X) \longrightarrow Y \times \boldsymbol{R}^{K} \quad \text { by } H(\mu)=(F(\mu), G(\mu)) .
$$

Then $H: M_{s}(X) \rightarrow H\left(M_{s}(X)\right)$ is quasi-perfect (cf. [16, Theorem 1.1]) and 1-1. Thus $M_{s}(X)$, being homeomorphic to a subspace of $Y \times \boldsymbol{R}^{K}$, is metrizable.

We consider $M_{s}(X)$ as a dense subset of $\boldsymbol{R}^{I}$, where $I$ is an algebraic basis of $C(X)$, and let $\mu_{0}$ be an element of $M_{s}(X)$ and $\left\{V_{n}\right\}$ a countable neighborhood base for $\mu_{0}$ in $M_{s}(X)$. Then $\left\{\mathrm{cl}_{R^{I}}\left(V_{n}\right)\right\}$ is a countable neighborhood base for $\mu_{0}$ in $\boldsymbol{R}^{I}$. It follows that $I$ is at most countable. Since no Banach space is of algebraic dimension $\boldsymbol{\aleph}_{0}$ (cf. [10, 14.32]), $I$ is finite. Therefore $X$ is finite.

(ii) $\Rightarrow$ (iii). Let $F_{n}=\left\{\mu \in M_{s}(X):\|\mu\| \leqq n\right\}$ for $n=1,2, \cdots$. Each 
$F_{n}$ is closed in $M_{s}(X)$ and $M_{s}(X)=\bigcup_{n=1}^{\infty} F_{n}$. Assume that $X$ is infinite. Then each $F_{n}$ has empty interior (cf. the proof of Theorem 16, Part II in [22]), so $M_{s}(X)$ is of the first category in itself and, in particular, it is not Cech complete.

3. Realcompactness. This section is concerned with the property of realcompactness in the spaces of measures. We recall that a completely regular space $X$ is realcompact if for every $y \in \beta X-X$ there is a continuous function $f: \beta X \rightarrow[0,1]$ such that $f(y)=0$ and $f(x)>0$ for all $x \in X$. The realcompactification of a space $X$ is denoted by $v X$; of course $X$ is realcompact if $X=v X$ (see [1]).

The following lemma is proved by Corson in [2] for the weak topology of a Banach space but essentially the same proof works in our case.

Lemma 3.1 (cf. [2, Lemma 9]). A vector subspace $M$ of $M(X)$ is realcompact if and only if every $\mu \in M(X)$ with the property

$(*)$ $\left\{\begin{array}{l}\text { for every countable } K \subset C(X) \\ \text { there is } \nu \in M \text { such that }\left.\mu\right|_{K}=\left.\nu\right|_{K}\end{array}\right.$ is, in fact, in $M$. More precisely,

$$
\cup M=\left\{\mu \in M(X): \mu \text { satisfies }\left({ }^{*}\right)\right\}
$$

with the weak topology.

This lemma implies immediately that $\cup M_{\sigma}(X)=M_{\sigma}(X)$, that is, $M_{\sigma}(X)$ is realcompact for any space $X$. Moreover, if $M_{1}, M_{2}$ are vector subspaces of $M(X)$ and $M_{1} \subset M_{2}$, then $v M_{1} \subset \cup M_{2} \subset M(X)$. Thus the relations between the spaces of measures imply the following diagram.

$$
\begin{aligned}
& M_{\sigma} \supset \cup M_{\tau} \supset \cup M_{t} \supset \cup M_{c} \\
& \cup \quad \cup \quad U \\
& M_{\tau} \supset M_{t} \supset M_{c} \text {. }
\end{aligned}
$$

Since a closed subset of a realcompact space is realcompact, in order that $M_{s}(X)$ (for $s=\tau, t$ or $c$ ) be realcompact a necessary condition is that $X$ be realcompact. Our purpose is to find when this condition is sufficient. First, we shall give another description of $v M_{s}(X)$ considering its elements as measures on $X$ rather than linear functionals on $C(X)$.

LEMMA 3.2. For every countable family $H$ of bounded Baire measurable functions on $X$ there is a countable set $K \subset C(X)$ such that, if $\mu$ and $\nu$ are $\sigma$-additive measures on $X$ and $\left.\mu\right|_{K}=\left.\nu\right|_{K}$, then 


$$
\int_{X} h d \mu=\int_{X} h d \nu \text { for all } h \in H
$$

Proof. Clearly it suffices to prove the lemma when $H$ consists of a single bounded Baire measurable function $h$. This is trivial when $h$ is continuous. In addition, if $\left\{h_{n}\right\}$ is a uniformly bounded sequence of Baire measurable functions which converges pointwise to some $h$ and the conclusion is true for every $h_{n}$, then, by the Lebesgue's dominated convergence theorem, the conclusion is true for $h$. This completes the proof.

THEOREM 3.3. Let $\mu \in M_{\sigma}(X)$. The following are equivalent (for $s=\tau, t$ or $c)$ :

(i) $\mu \in \cup M_{s}(X)$;

(ii) for every countable family $H$ of bounded Baire measurable functions, there is $\nu \in M_{s}(X)$ such that $\int_{X} h d \mu=\int_{X} h d \nu$ for all $h \in H$;

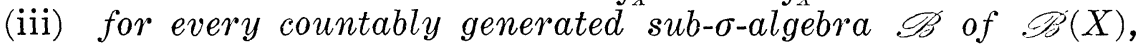
there is $\nu \in M_{s}(X)$ such that $\mu(B)=\nu(B)$ for all $B \in \mathscr{B}$;

(iv) for every continuous function $f$ from $X$ into a separable metric space, there is $\nu \in M_{s}(X)$ such that $f_{*}(\mu)=f_{*}(\nu)$.

\section{Proof. (i) $\Longrightarrow$ (ii) is immediate from Lemmas 3.1 and 3.2.}

(ii) $\Rightarrow$ (iii). Let $\mathscr{B}$ be a countably generated sub- $\sigma$-algebra of $\mathscr{B}(X)$. By [11, I, $\S 2$, Theorem 5] there is $f: X \rightarrow \boldsymbol{R}$ Baire measurable such that $\mathscr{B}=f^{-1}(\mathscr{B}(\boldsymbol{R}))$. Let $\left\{V_{n}\right\}$ be a countable base for the topology of $\boldsymbol{R}$. We may suppose that $\left\{V_{n}\right\}$ is closed under finite unions. From (ii) it follows that there is $\nu \in M_{s}(X)$ such that $\mu\left(f^{-1}\left(V_{n}\right)\right)=\nu\left(f^{-1}\left(V_{n}\right)\right)$ for all $n$, so $f_{*}(\mu)=f_{*}(\nu)$. Therefore $\mu(B)=$ $\nu(B)$ for all $B \in \mathscr{B}$.

(iii) $\Rightarrow$ (iv). This is trivial since $f^{-1}(\mathscr{B}(Y))$ is countably generated.

(iv) $\Rightarrow$ (i). Let $K=\left\{f_{n}: n \in N\right\}$ be a countable subset of $C(X)$. We define

$$
f: X \longrightarrow R^{N} \quad \text { by } \quad f(x)=\left(f_{n}(x)\right)_{n \in N} \text {. }
$$

By (iv), there is $\nu \in M_{s}(X)$ such that $f_{*}(\mu)=f_{*}(\nu)$. It follows that $\mu$ and $\nu$ coincide on $f_{n}^{-1}(\mathscr{B}(\boldsymbol{R}))$, so $\mu\left(f_{n}\right)=\nu\left(f_{n}\right)$ for all $n$. From Lemma 3.1 it follows that $\mu \in \cup M_{s}(X)$.

REMARK. It is clear that Theorem 3.3 is valid for any vector subspace $M$ of $M_{o}(X)$ in the place of $M_{s}(X)$. So direction (i) $\Rightarrow$ (iii) implies that the space $M_{p}(X)$ of perfect measures on a completely regular space $X$ (see [14]) is always realcompact. Since $M_{p} \supset M_{t}$ we have $M_{\sigma} \supset M_{p} \supset \cup M_{t}$.

Some well-known properties of the spaces of measures remain 
valid for their realcompactifications.

Corollary 3.4. If $\mu \in \cup M_{s}(X)$ and $A \in \mathscr{B}(X)$, then the measure $\mu_{A}$ defined by

$$
\mu_{A}(B)=\mu(A \cap B), \quad B \in \mathscr{B}(X)
$$

is in $\cup M_{s}(X)$ (hence $\mu^{+}, \mu^{-}$and $|\mu|$ are also in $v M_{s}(X)$ ) for $s=\tau, t$ or c.

Corollary 3.5. If $f: X \rightarrow Y$ is continuous, then $f_{*}\left(\cup M_{s}(X)\right) \subset$ $\cup M_{s}(Y)$ for $s=\tau, t$ or $c$.

Proposition 3.6. If $X$ is realcompact, then $M_{c}(X)$ is realcompact.

Proof. Let $\mu$ be in $\cup M_{c}(X)$ and $S_{\mu}$ be the support of the corresponding measure $\bar{\mu}$ on $\beta X$. It suffices to show that $S_{\mu} \subset X$. Let $y \in \beta X-X$. Since $X$ is realcompact, there is $f: X \rightarrow[0,1]$ continuous such that $\bar{f}(y)=0$ and $\bar{f}(x)>0$ for all $x \in X$, where $\bar{f}: \beta X \rightarrow[0,1]$ is the continuous extension of $f$. By Theorem 3.3 (iv), there is $\nu \in M_{c}(X)$ such that $f_{*}(\mu)=f_{*}(\nu)$. It follows that 0 is not in the support of $f_{*}(\mu)$, hence $y \notin S_{\mu}$.

The analogue of Proposition 3.6 is not correct for the spaces $M_{\tau}$ and $M_{t}$. First, we prove the following.

Proposition 3.7. For any cardinal $\alpha, \cup M_{t}\left(\boldsymbol{R}^{\alpha}\right)=\cup M_{\tau}\left(\boldsymbol{R}^{\alpha}\right)=$ $M_{\sigma}\left(\boldsymbol{R}^{\alpha}\right)$.

Proof. Let $\mu \in M_{\sigma}\left(\boldsymbol{R}^{\alpha}\right)$ and $f: \boldsymbol{R}^{\alpha} \rightarrow Y$ a continuous function, where $Y$ is a separable metric space. By Theorem 3.3 (iv), it suffices to show that there is $\nu \in M_{t}\left(\boldsymbol{R}^{\alpha}\right)$ such that $f_{*}(\mu)=f_{*}(\nu)$.

It is known (cf. [20]) that $f$ is determined by countably many coordinates, that is, there is $A \subset \alpha$ countable such that $f=g \circ p$, where $p: \boldsymbol{R}^{\alpha} \rightarrow \boldsymbol{R}^{A}$ is the projection and $g$ is continuous. Let $q: \boldsymbol{R}^{A} \rightarrow \boldsymbol{R}^{\alpha}$ be a continuous function such that $p \circ q$ is the identity function of $\boldsymbol{R}^{A}$. Since $\boldsymbol{R}^{A}$ is separable complete metrizable, the measure $p_{*}(\mu)$ is tight. If $\nu=q_{*}\left(p_{*}(\mu)\right)$, then $\nu \in M_{t}\left(\boldsymbol{R}^{\alpha}\right)$ and, for every $A \in \mathscr{B}(Y)$, we have

$$
\begin{aligned}
f_{*}(\mu)(A) & =p_{*}(\mu)\left(g^{-1}(A)\right) \\
& =p_{*}\left(q_{*}\left(p_{*}(\mu)\right)\right)\left(g^{-1}(A)\right) \\
& =p_{*}(\nu)\left(g^{-1}(A)\right) \\
& =f_{*}(\nu)(A) .
\end{aligned}
$$

The above proposition in conjunction with some known results yields the following (a) and (b). 
(a) $M_{\tau}\left(\boldsymbol{R}^{c}\right)$ and $M_{t}\left(\boldsymbol{R}^{c}\right)$ (where $c$ is the continuum) are not realcompact, although $\boldsymbol{R}^{c}$ is realcompact. Indeed, it is known that $M_{\sigma}\left(\boldsymbol{R}^{c}\right) \neq M_{\tau}\left(\boldsymbol{R}^{c}\right)$ (see [12] or [17]) and, of course, $M_{\sigma}\left(\boldsymbol{R}^{c}\right) \neq M_{t}\left(\boldsymbol{R}^{c}\right)$.

(b) The proposition " $M_{\tau}\left(\boldsymbol{R}^{\aleph_{1}}\right)$ is realcompact" is undecidable in ZFC, although $\boldsymbol{R}^{\aleph_{1}}$ is realcompact. Indeed, Fremlin [4] showed that if we assume Martin's Axiom and the negation of the continuum hypothesis, then $M_{\sigma}\left(\boldsymbol{R}^{\aleph_{1}}\right)=M_{\tau}\left(\boldsymbol{R}^{\aleph_{1}}\right)$. On the other hand, if we assume the continuum hypothesis, then $M_{\sigma}\left(\boldsymbol{R}^{\aleph_{1}}\right) \neq M_{\tau}\left(\boldsymbol{R}^{\aleph_{1}}\right)$ by the result mentioned in (a).

We notice that it is consistent with the negation of the continuum hypothesis for $M_{\tau}\left(\boldsymbol{R}^{\boldsymbol{N}_{1}}\right)$ not to be realcompact (see [9]). Also, $M_{t}\left(\boldsymbol{R}^{\aleph_{1}}\right)$ is not realcompact whether or not $c=\boldsymbol{\aleph}_{1}$ because $M_{\sigma}\left(\boldsymbol{R}^{\aleph_{1}}\right) \neq$ $M_{t}\left(\boldsymbol{R}^{\aleph_{1}}\right)$ ([4, Remark (b)]).

A space $X$ with the countable chain condition is realcompact if and only if $X$ is topologically complete (cf. [2, Lemma 8]). Therefore Lemma $2.8(\mathrm{i})$ and (b) above yield that the proposition " $M_{\tau}\left(\boldsymbol{R}^{\aleph_{1}}\right)$ is topologically complete" is also undecidable in ZFC. This shows that topological completeness is not a purely topological property. See also $[8$, p. 12].

In the next theorem we show that realcompactness devolves from $X$ to $M_{\tau}(X)$ and $M_{t}(X)$, if $X$ is metrizable. We recall that, by (a special form of) Katetov's theorem (cf. [1, Theorem 6.2]), a metric space $X$ is realcompact if and only if every closed discrete subset of $X$ has non-(Ulam-) measurable cardinal. Also, a $\sigma$-additive measure on $X$ is $\tau$-additive if and only if it is supported by a closed separable subset.

THeOREM 3.8. If $X$ is a realcompact metric space, then $M_{\tau}(X)$ and $M_{t}(X)$ are realcompact.

Proof. Let $\mu \in \cup M_{\tau}(X), \mu \geqq 0$. By Corollary 3.4 , in order to prove that $M_{\tau}(X)$ is realcompact, it suffices to show that $\mu \in M_{\tau}(X)$. Let $\mathscr{U}$ be the family of all open sets of $\mu$-measure zero. The set $X-\cup \mathscr{C}$ satisfies the countable chain condition, so it is separable and it is enough to show that $\mu(\cup \mathscr{C})=0$. By Stone's theorem there is a $\sigma$-discrete refinement $\mathscr{V}=\bigcup_{n=1}^{\infty} \mathscr{O}_{n}$, each $\mathscr{V}_{n}$ discrete and $\cup \mathscr{O}=$ $\cup \mathscr{U}$. If we suppose that $\mu(\cup \mathscr{U})>0$, then $\mu\left(\cup \mathscr{V}_{n}\right)>0$ for some $n$. Fix such an $n$.

For each $V \in \mathscr{V}_{n}$ we choose a point $x_{V} \in V$ and set $T=\left\{x_{V}: V \in \mathscr{V}_{n}\right\}$. Let $f: X \rightarrow T$ with $\left.f\right|_{V}=x_{V}$ for all $V \in \mathscr{V}_{n}$ and $\left.f\right|_{X-U \mathscr{V}_{n}}$ constant. The cardinal of $T$ if nonmeasurable because $T$ is closed and discrete in $X$ which is assumed to be realcompact. Thus, the image measure $\kappa=$ $f_{*}\left(\mu_{\cup \mathscr{V}_{n}}\right)$ which is defined on all subsets of $T$ and vanishes on singletons is non-atomic. It follows (cf. $[14,2.1]$ ) that there is $g: T \rightarrow$ 
$[0,1]$ such that $g_{*}(\kappa)=\alpha \cdot \lambda$, where $\alpha=\mu\left(\cup \mathscr{V}_{n}\right)>0$ and $\lambda$ is the Lebesgue measure on the Baire sets of $[0,1]$. If $h=g \circ f$, then $h_{*}\left(\mu_{\cup \mathscr{V}_{n}}\right)=\alpha \cdot \lambda$. Since $h^{-1}(\mathscr{B}([0,1]))$ is countably generated and $\mu_{\cup \mathscr{V}_{n}} \in \cup M_{\tau}(X)$ (Corollary 3.4), there is $\nu \in M_{\tau}(X)$ such that $h_{*}\left(\mu_{\cup v_{n}}\right)=$ $h_{*}(\nu)$ (Theorem 3.3). By the $\tau$-additivity, $\nu$ is supported by $\cup \mathscr{W} \cup$ $\left(X-\cup \mathscr{\mathscr { V }}_{n}\right)$ for some countable $\mathscr{W}^{-} \subset \mathscr{\mathscr { V }}_{n}$. But $h\left(\cup \mathscr{W} \cup\left(X-\cup \mathscr{C}_{n}\right)\right)$ is countable, so $h_{*}(\nu)$ is supported by a countable set. This is a contradiction since $h_{*}(\nu)=\alpha \cdot \lambda$.

To show that $M_{t}(X)$ is realcompact let $\mu \in \cup M_{t}(X)$. Then $\mu \in$ $v M_{\tau}(X)=M_{\tau}(X)$ by the above. So, $\mu$ is supported by a closed separable set $S$. Since $\mathscr{B}(S)$ is countably generated, it follows from Theorem 3.3, that $\mu \in M_{t}(X)$.

Theorem 3.8 at least for the space of $\tau$-additive measures remains valid when $X$ is paracompact. Indeed, the only properties of metric spaces which are actually used are paracompactness and that every open set is a Baire set. However, the latter is not essential (cf. the proof of Theorem 5.10 in [14]). In any case, this result will be deduced as corollary of the next theorem. First we give some notations and definitions related to the notion of continuous pseudometric.

We denote by $\mathscr{D}$ the family of all continuous pseudometrics on a completely regular space $X$. If $d \in \mathscr{D}$, let $\pi_{d}$ be the natural projection from $X$ onto the corresponding metric space $X_{d}$. A subset $A$ of $X$ is $d$-discrete if there exists $\varepsilon>0$ such that $d(x, y) \geqq \varepsilon$ for all $x, y \in A, x \neq y$. As in [15], we say that $X$ is a $D_{0}$-space if, for every $d \in \mathscr{D}$, all $d$-discrete subsets of $X$ have nonmeasurable cardinal. It follows from Katetov's theorem that $X$ is a $D_{0}$-space if and only if $X_{d}$ is realcompact for every $d \in \mathscr{D}$ (cf. [15]).

The space $M_{u}(X)$ of the $u$-additive measures on $X$ can be defined as follows: A measure $\mu$ on $X$ is in $M_{u}(X)$ if $\left(\pi_{d}\right)_{*}(\mu) \in M_{\tau}\left(X_{d}\right)$ for all $d \in \mathscr{D}$. We have $M_{\sigma}(X) \supset M_{u}(X) \supset M_{\tau}(X)$ and $M_{u}(X)=M_{\tau}(X)$ whenever $X$ is paracompact (see [21]).

THEOREM 3.9. For any space $X$ the following are equivalent:

(i) $M_{u}(X)$ is realcompact;

(ii) $X$ is a $D_{0}$-space.

Proof. (i) $\Rightarrow$ (ii). The topological completion $\theta X$ of $X$ (see [1] for the definition of this term) is homeomorphic to the closed subset of $M_{u}(X)$ of the 2-valued $u$-additive measures, hence $\theta X$ is realcompact. For every $d \in \mathscr{D}$, let $\bar{\pi}_{d}: \theta X \rightarrow X_{d}$ be the continuous extension of $\pi_{d}$. By the Katetov's theorem $X_{d}$ is realcompact as a metrizable continuous image of a realcompact space. 
(ii) $\Rightarrow$ (i). Let $\mu \in \cup M_{u}(X)$ and $d \in \mathscr{D}$. As in Corollary 3.5 we have $\left(\pi_{d}\right)_{*}(\mu) \in v M_{u}\left(X_{d}\right)$. But $v M_{u}\left(X_{d}\right)=v M_{\tau}\left(X_{d}\right)=M_{\tau}\left(X_{d}\right)$ by Theorem 3.8. Therefore $\mu \in M_{u}(X)$.

Since $M_{u}(X)=M_{\tau}(X)$ for paracompact spaces and realcompact spaces are $D_{0}$-spaces, we have:

COROLlaRY 3.10. If $X$ is a realcompact paracompact space, then $M_{\tau}(X)$ is realcompact.

I don't know if the analogue of this corollary is true for the space of tight measures. (However, it is true for paracompact $M$ spaces).

Proposition 3.11. (i) $M_{u}^{+}(X)$ is topologically complete for any space $X$; (ii) $M_{c}^{+}(X)$ is topologically complete if (and only if) $X$ is topologically complete.

Proof. (i ) For every $d \in \mathscr{D}$, the function $\left(\pi_{d}\right)_{*}: M^{+}(X) \rightarrow M^{+}\left(X_{d}\right)$ is continuous and we have

$$
M_{u}^{+}(X)=\bigcap_{d \in \mathscr{D}}\left(\pi_{d}\right)_{*}^{-1}\left(M_{\tau}^{+}\left(X_{d}\right)\right) .
$$

The spaces $M^{+}(X)$ and $M_{-}^{+}\left(X_{d}\right)$ are topologically complete since

$$
M^{+}(X)=\bigcup_{n=1}^{\infty}\left\{\mu \in M^{+}(X): \mu(X) \leqq n\right\}
$$

is $\sigma$-compact and $M_{-}^{+}\left(X_{d}\right)$ is metrizable (Theorem 1.2). It follows that $\left(\pi_{d}\right)_{*}^{-1}\left(M_{*}^{+}\left(X_{d}\right)\right)$ is topologically complete for every $d \in \mathscr{D}$, so $M_{u}^{+}(X)$ is topologically complete as an intersection of such spaces (see [1, 1.8 and 1.10]).

(ii) If $X$ is topologically complete, for every $y \in \beta X-X$ there is $d \in \mathscr{D}$ such that $\bar{\pi}_{d}(y) \in \beta X_{d}-X_{d}$, where $\bar{\pi}_{d}: \beta X \rightarrow \beta X_{d}$ is the continuous extension of $\pi_{d}$ ([1, Theorems 3.2 and $\left.4.4(\mathrm{~d})\right]$ ). As in Proposition 3.6 we can show that a measure $\mu \in M^{+}(X)$ has compact support if and only if $\left(\pi_{d}\right)_{*}(\mu) \in M_{c}^{+}\left(X_{d}\right)$ for every $d \in \mathscr{D}$. Since $M_{c}^{+}(X) \subset M_{-}^{+}\left(X_{d}\right)$ is metrizable, using similar arguments as in (i), we find that $M \sim(X)$ is topologically complete.

REMARKS. Proposition 3.11 (ii) is not much different from Proposition 3.6 since a topologically complete space of nonmeasurable cardinal is realcompact by the Shirota theorem ([1, Theorem 6.3]). However, the restriction to the subset of positive measures is necessary. Indeed, if $X$ is a discrete space then $M_{c}^{+}(X)$ is topologically complete (Prop. 3.11(ii)), while $M_{c}(X)$ is topologically complete if 
and only if the cardinal of $X$ is nonmeasurable (Lemma 2.8 (i) and Prop. 3.6).

A similar argument shows that Proposition 3.11 (i) is not correct for $M_{u}(X)$.

\section{REFERENCES}

1. W. W. Comfort and S. Negrepontis, Continuous Pseudometrics, Marcel Dekker, New York, 1975.

2. H. H. Corson, The weak topology of a Banach space, Trans. Amer. Math. Soc., 101 (1961), 1-15.

3. R. Engelking, Outline of general topology, North Holland, Amsterdam, \& Polish Scientific Publishers, Warzawa, 1968.

4. D. H. Fremlin, Uncountable products of $R$ can be almost Lindelöf, Manuscripta Math., 22 (1977), 77-85.

5. E. Granirer, On Baire measures on D-topological spaces, Fund. Math., 60 (1967), $1-22$.

6. W. Grömig, On a weakly closed subset of the space of $\tau$-smooth measures, Proc. Amer. Math. Soc., 43 (1974), 397-401.

7. P. R. Halmos, Measure Theory, Van Nostrand, Princeton, 1960.

8. R. G. Haydon, On compactness in spaces of measures and measurecompact spaces, Proc. London Math. Soc., 29 (1974), 1-16.

9. S. H. Hechler, On $N \aleph_{1}$ and the almost Lindelöf property, Proc. Amer. Math. Soc., 52 (1975), 353-355.

10. E. Hewitt and K. Stromberg, Real and Abstract Analysis, Springer-Verlag, 1965.

11. J. Hoffman-Jørgensen, The Theory of Analytic Spaces, Various Publication Series

10, Aarchus, 1970.

12. J. H. B. Kemperman and D. Maharam, $R^{c}$ is not almost Lindelöf, Proc. Amer. Math. Soc., 24 (1970), 772-773.

13. J. D. Knowles, Measures on topological spaces, Proc. London Math. Soc., 17 (1967), $139-156$.

14. G. Koumoullis, On perfect measures, Trans. Amer. Math. Soc., 264 (1981), 521-537. 15. - Perfect, u-additive measures and strict topologies, to appear in Illinois J. Math.

16. E. Michael, A theorem on perfect maps, Proc. Amer. Math. Soc., 28 (1971), 633-634. 17. W. Moran, The additivity of measures on completely regular spaces, J. London Math. Soc., 43 (1968), 633-639.

18. K. Morita, A survey of the theory of M-spaces, Gen. Topology Appl., 1 (1971), 49-55.

19. S. E. Mosiman and R.F. Wheeler, The strict topology in a completely regular setting: Relations to topological measure theory, Canad. J. Math., 24 (1972), 873-890.

20. K. A. Ross and A. H. Stone, Products of separable spaces, Amer. Math. Monthly, 71 (1964), 398-403.

21. F.D. Sentilles and R.F. Wheeler, Linear functionals and partitions of unity in $C_{b}(X)$, Duke Math. J., 41 (1974), 483-496.

22. V.S. Varadarajan, Measures on topological spaces, Amer. Math. Soc. Transl., 48 (1965), 161-228.

23. M. D. Weir, Hewitt-Nachbin spaces, North Holland, Amsterdam, 1975.

Received June 20, 1980. 



\section{PACIFIC JOURNAL OF MATHEMATICS}

\section{EDITORS}

DoNALD BABBITT (Managing Editor)

University of California

Los Angeles, CA 90024

Hugo Rossi

University of Utah

Salt Lake City, UT 84112

C. C. MOORE and ANDREw OGG

University of California

Berkeley, CA 94720
J. DugundJI

Department of Mathematics

University of Southern California

Los Angeles, CA 90007

R. FinN and J. Milgram

Stanford University

Stanford, CA 94305

ASSOCIATE EDITORS
R. ARENS
E. F. BECKENBACH
B. H. NeumanN
F. WOLF
K. YoshidA

\section{SUPPORTING INSTITUTIONS}

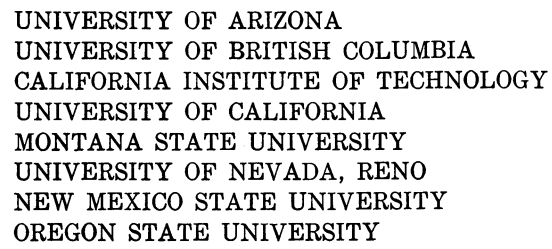

UNIVERSITY OF ARIZONA

UNIVERSITY OF BRITISH COLUMBIA

CALIFORNIA INSTITUTE OF TECHNOLOGY

UNIVERSITY OF CALIFORNIA

MONTANA STATE UNIVERSITY

UNIVERSITY OF NEVADA, RENO

NEW MEXICO STATE UNIVERSITY OREGON STATE UNIVERSITY

\author{
UNIVERSITY OF OREGON \\ UNIVERSITY OF SOUTHERN CALIFORNIA \\ STANFORD UNIVERSITY \\ UNIVERSITY OF HAWAII \\ UNIVERSITY OF TOKYO \\ UNIVERSITY OF UTAH \\ WASHINGTON STATE UNIVERSITY \\ UNIVERSITY OF WASHINGTON
}

The Supporting Institutions listed above contribute to the cost of publication of this Journal, but they are not owners or publishers and have no responsibility for its content or policies.

Mathematical papers intended for publication in the Pacific Journal of Mathematics should be in typed form or offset-reproduced, (not dittoed), double spaced with large margins. Please do not use built up fractions in the text of the manuscript. However, you may use them in the displayed equations. Underline Greek letters in red, German in green, and script in blue. The first paragraph or two must be capable of being used separately as a synopsis of the entire paper. Please propose a heading for the odd numbered pages of less than 35 characters. Manuscripts, in triplicate, may be sent to any one of the editors. Please classify according to the scheme of Math. Reviews, Index to Vol. 39. Supply name and address of author to whom proofs should be sent. All other communications should be addressed to the managing editor, or Elaine Barth, University of California, Los Angeles, California, 90024.

50 reprints to each author are provided free for each article, only if page charges have been substantially paid. Additional copies may be obtained at cost in multiples of 50 .

The Pacific Journal of Mathematics is issued monthly as of January 1966. Regular subscription rate: $\$ 102.00$ a year (6 Vols., 12 issues). Special rate: $\$ 51.00$ a year to individual members of supporting institutions.

Subscriptions, orders for numbers issued in the last three calendar years, and changes of address shoud be sent to Pacific Journal of Mathematics, P.O. Box 969, Carmel Valley, CA 93924, U.S.A. Old back numbers obtainable from Kraus Periodicals Co., Route 100, Millwood, NY 10546.

\footnotetext{
PUBLISHED BY PACIFIC JOURNAL OF MATHEMATICS, A NON-PROFIT CORPORATION

Printed at Kokusai Bunken Insatsusha (International Academic Printing Co., Ltd.). 8-8, 3-chome, Takadanobaba, Shinjuku-ku, Tokyo 160, Japan.
} 


\section{Pacific Journal of Mathematics}

\section{Vol. 96, No. 2 December, 1981}

Gerald A. Beer, A natural topology for upper semicontinuous functions and

a Baire category dual for convergence in measure $\ldots \ldots \ldots \ldots \ldots \ldots 251$

Georgia Benkart and J. Marshall Osborn, An investigation of real

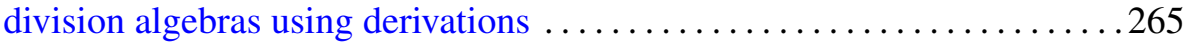

Donald Ian Cartwright and John R. McMullen, A structural criterion for the existence of infinite Sidon sets ........................ 301

Philip Hanlon, The fixed-point partition lattices $\ldots \ldots \ldots \ldots \ldots \ldots \ldots \ldots$

Eric Hayashi, The spectral density of a strongly mixing stationary Gaussian process

Chung-Wu Ho and Charles E. Morris, Jr., A graph-theoretic proof of

Sharkovsky's theorem on the periodic points of continuous functions . ...361

Sara Hurvitz, The automorphism groups of spaces and fibrations ....... 371

Atsushi Inoue, Schoichi Ota and Jun Tomiyama, Derivations of operator algebras into spaces of unbounded operators . .................. 389

Wolfgang B. Jurkat and Gary Sampson, On weak restricted estimates and endpoint problems for convolutions with oscillating kernels. I ........ 405

Georgios Koumoullis, Some topological properties of spaces of

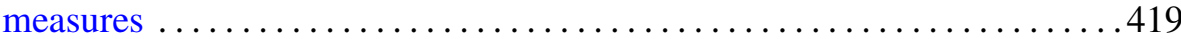

Wen Hsiung Lin, Algebraic Kahn-Priddy theorem ................. 435

Michael John McAsey, Invariant subspaces of nonselfadjoint crossed

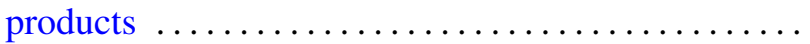

Justin Peters, Entropy of automorphisms on L.C.A. groups

Saburou Saitoh, A characterization of the adjoint $L$-kernel of Szegó type 